\title{
Capacidades de innovación en los emprendedores turísticos: Un enfoque de género
}

\section{Innovation capabilities in tourism entrepreneurs: A gender approach}

\author{
Luis Oswaldo Manosalvas Vaca \\ https://orcid.org/0000-0002-4659-8090 \\ Carlos Aníbal Manosalvas Vaca \\ https://orcid.org/0000-0002-7521-069X \\ Universidad Estatal Amazónica, Ecuador \\ Viviana Catalina Solís Morejón \\ https://orcid.org/0000-0002-5961-7406 \\ Escuela Superior Politécnica del Chimborazo, Ecuador \\ Johana Karen Pesantez Quintanilla \\ https://orcid.org/0000-0002-1027-56 \\ Investigadora independiente, Ecuador
}

Autor por correspondencia: lmanosalvas@uea.edu.ec; cmanosalvas@uea.edu.ec; viviana.solis@espoch.edu.ec; johana_k89@hotmail.com

Fecha de recepción: 10 de marzo de 2020 - Fecha de aceptación: 29 de abril de 2020

\section{Resumen}

El afán de crear nuevos y mejores productos - servicios a través de ideas y procesos innovadores se ha incrementado en los últimos años, desarrollando capacidades en una persona para mejorar su situación en el ámbito emprendedor y permanecer dentro del mercado sobresaliendo de entre otros negocios. En esta investigación se analizó las capacidades de innovación de los emprendedores turísticos, la muestra fue desarrollada en la región Amazónica del Ecuador, específicamente en la Provincia de Pastaza, el objetivo principal del estudio fue determinar si existen diferencias en el género en lo que respecta a capacidades de innovación, la investigación se desarrolló a través de una metodología cuantitativa mediante un paradigma deductivo, el tipo de investigación fue documental y de campo, método no experimental, transversal y descriptivo, el instrumento de medición fue sometido a un análisis de "Confiabilidad de Cronbach" y se realizó una prueba " $t$ " de student para estimar una diferencia significativa entre las medias de dos grupos (femenino y masculino). Los resultados muestran una demografía con una población adulta con predominancia del género femenino, también, se obtuvieron datos positivos en relación a la capacidad de innovación donde los emprendedores tienen habilidades para emprender y manejar con ingenio diversas situaciones, se identificó que no existe diferencia significativa entre los dos géneros de acuerdo a las habilidades que cada uno posee.

Palabras clave: capacidades; innovación; emprendedores; turismo; género 


\begin{abstract}
The desire to create new and better products - services through innovative ideas and processes has increased in recent years, developing capacities in a person to improve their situation in the entrepreneurial field and remain within the market, standing out from among other businesses. This research aims to analyze the innovation capabilities of tourism entrepreneurs, the sample was developed in the Amazon region of Ecuador, specifically in the Province of Pastaza, the main objective of the study was to determine if there are differences in gender in regard to innovation capabilities, the research was developed through a quantitative methodology through a deductive paradigm, the type of research was documentary and field, nonexperimental, transversal and descriptive method, the measuring instrument was subjected to an analysis of "Reliability of Cronbach "and a student" $t$ "test was performed to estimate a significant difference between the means of two groups (female and male). The results show demography with an adult population with a predominance of the female gender, also, positive data were obtained in relation to the capacity for innovation where entrepreneurs have skills to undertake and manage various situations witfully, it was identified that there is no significant difference between the two genres according to the abilities that each one possesses.
\end{abstract}

Keywords: capabilities; innovation; entrepreneurs; tourism; gender.

\title{
Introducción
}

Hoy en día el Turismo es una de las actividades que contiene variantes importantes para la superación de un país, generando y reactivando la economía a través de distintas acciones que se desarrollan dentro del lugar, con la aparición de más puestos de trabajo e infraestructuras representativas, factor fundamental para el incremento de ingresos en bien de la población, con la renovación y el mantenimiento de los destinos turísticos para el uso correcto (Bembibre, 2011). La innovación es un punto clave para el surgimiento de etapas dentro del Turismo en relación a los diferentes procesos que conllevan a captar nuevas y mejores tendencias ya que en la actualidad existe un entorno muy dinámico en donde hay que identificar oportunidades para poder anticiparse a las necesidades y superar las expectativas de los visitantes; pues, la forma de trabajo de los emprendedores que brindan el servicio turístico debe cambiar según lo que emana la competencia en el mercado; las capacidades de innovación también son pilares principales para la transformación en la calidad de gestión en asistencia a la prestación de servicios turísticos que influye de manera favorable en cada situación presentada (Sustentur, 2015).

El principal rubro económico del cantón Pastaza es la agricultura, aunque Pastaza es considerado como lugar turístico, siendo la segunda fuente económica (Andino, 2016). Sin embargo, a pesar que la Cámara Pequeña de Turismo de Pastaza CAPTUR considera que existe innovación en los emprendimientos turísticos, en la realidad no se evidencia tales actividades de innovación, por lo cual, se considera que las empresas están fracasando. Existen varias microempresas turísticas que necesitan el apoyo pertinente para la variación de los servicios en dependencia de la situación actual, así como la presentación de innovadoras tendencias, la falta de innovación en los emprendimientos turísticos presenta una baja consolidación en el entorno competitivo, las similitudes de los negocios ocasionan el cierre del emprendimiento a corto tiempo debido a la falta de incorporación de ideas propias y flexibles acorde al sector (Liévano, Vargas, Pico y Pérez, 2018). En esta investigación se está evaluando las capacidades de innovación de los emprendedores turísticos del cantón Pastaza ubicada en la región amazónica del Ecuador con un enfoque de género, para conocer el grado de efectividad que poseen en relación a las nueve dimensiones como la motivación, 
proactividad, auto concepto de innovación, redes, comunicación persuasiva, trabajo en equipo, cognición creativa, intención de innovar y toma de riesgos, que complementan al tema en cuestión, las cuales a través de un instrumento de medición se analizó la muestra seleccionada, empezando con los datos demográficos y concluyendo con un análisis que represente la forma o estructura productiva que realiza el emprendedor, analizando si existe diferencias significativas entre hombres y mujeres o simplemente sus igualdad en la capacidad de innovación que cada uno de ellos tienen para tomar las riendas de sus negocios (Putruele, 2017).

\section{Turismo en el mundo}

\section{Marco teórico}

El Turismo y la Innovación se encuentran en grandes cambios en el mundo por el incremento de la actividad turística, las empresas tienden a alcanzar altos niveles de competitividad llegando a tener una búsqueda de innovación y creatividad mediante programas y estrategias que conlleva al Turismo a un nuevo modelo turístico, en la cual, el internet es una de las herramientas que facilita a los turistas a obtener de manera rápida la información de los lugares turísticos al que desea viajar, por lo que las empresas turísticas se adaptan al nuevo modelo como la tecnología de la información y las comunicaciones, la innovación de algunas herramientas y programas turísticos ayuda a que el servicio sea personalizado (López, Palomeque \& Fadul,2016).

En los últimos años, el Turismo a nivel mundial ha ido progresando hasta que ha obtenido grandes cambios gracias a la innovación y creatividad desarrollada en todas partes, de tal forma, es una de las acciones importantes para el crecimiento económico, desarrollo cultural de la población y a la vez es una de las actividades que genera mayor empleo, permitiendo que la población tenga una mejor calidad de vida, también abarca las diferentes actividades que conlleva a la creación de nuevas empresas turísticas e innovadoras como: alojamiento, transporte y operación turística, considerando una de las funciones más dinámicas en la que el turista goza de los servicios que brinda la actividad turística (García, 2014). El Turismo además de ser una de las actividades que genera economía a la sociedad, también ayuda a recuperar las tradiciones, cultura y medio ambiente de un entorno para aprovechar de manera sostenible y oportuna, obteniendo efectos positivos en el lugar a realizar las actividades turísticas, así es como, el Turismo viene a ser una estrategia primordial que incentiva a los organismos internacionales a controlar y regular la actividad turística a través de una normativa que determina un aporte necesario dentro de esta acción cumpliendo con los aspectos de mayor relevancia (Juárez \& Cebrián, 2016).

\section{El Turismo en el Ecuador}

En las últimas décadas el Turismo es uno de las actividades que dinamiza la economía y es indispensable en el fortalecimiento de la sociedad actual, de esta manera el Turismo se ha convertido en uno de los ejes importantes del crecimiento de la economía del Ecuador a corto y mediano plazo, tiende a mejorar y prestar servicios de buena calidad para llegar a ser competitivo con miras a un modelo de desarrollo sostenible de los diferentes atractivos turísticos, el Ministerio de Turismo (MINTUR) y la Dirección de calidad basándose en las competencias correspondientes busca una nueva estrategia para la implementación de un sistema de calidad generando y adoptando una cultura de excelencia para posicionar a 
Ecuador adelante de los demás países y ser potencia turística en el mercado nacional e internacional (Samson, 2015).

Ecuador es uno de los países que está apostando a la actividad turística el cual posee una variedad de atractivos turísticos que ofrece al mundo gracias a su innovación, de tal manera, se ha elevado el ingreso de turistas al país, por ende, existe ingresos económicos e inversiones. Ecuador tiende a desarrollar actividades de innovación en temáticas de: innovación en la formación de profesionales turísticos, innovación en el desarrollo de productos turísticos y en destinos turísticos (López, Palomeque \& Fadul, 2016).

\section{Innovación}

La innovación es una de las herramientas claves, hablar hoy sobre el tema es inevitable por lo que se involucra en diversos aspectos como en las empresas, políticas y academia. Para las empresas la innovación es fundamental en relación a su supervivencia y sostenibilidad para permanecer en el mercado ya que hoy en día se encuentran compitiendo para sobresalir y ser mejor que las demás, en cuanto a la política el estado estimula ciertos aspectos para optimizar y realizar un modelo de desarrollo económico, en cierta forma, pretende la competitividad del país mediante diseños de las políticas públicas (Restrepo, 2013). Con respecto a la academia en las universidades, la innovación es fundamental porque estas instituciones son fuentes de oportunidad que generan mecanismos de información científica, además aparecen nuevas ideas y modelos de emprendimientos en los estudiantes en la que adquieren e intercambian conocimientos (Selznik \& Mayhew, 2017).

De acuerdo a los estudios ya realizados, la innovación tiende a clasificar en innovaciones radicales e innovaciones incrementales. La primera se basa en realizar nuevos productos que a simple vista se diferencien de otros y que sean llamativos. La segunda trata sobre los productos ya existentes que toma la iniciativa de modificar y mejorar el tamaño de dicho producto (Ramírez \& Vega, 2015). La incorporación de la innovación en los diferentes aspectos es una estrategia que da paso a una nueva oportunidad de realizar y crear nuevos productos y/o servicios que favorecen a las empresas e instituciones que desean mejorar para sobresalir en este mundo cambiante llena de características innovadoras (Arenas, 2017). La innovación es importante en el Turismo ya que interviene en la creación de nuevos productos y servicios, además estas se adaptan a las nuevas tecnologías, pues los individuos tienen la capacidad de innovar diferentes modelos de negocios turísticos mediante algunos tipos de estrategias, facilitan a las empresas para que así ellas puedan competir en el mercado empresarial por ello existe un crecimiento económico que ayuda a las organizaciones, empresas y al país en general (Álvarez, Andrade, Bravo, Rodríguez \& Govea, 2016).

\section{Innovación en el Turismo}

Es de gran importancia la innovación en el sector turístico ya que es un instrumento que facilita el alcance del crecimiento perdurable y sostenible de las empresas turísticas además permite mejorar la competitividad de las empresas en el mercado en la que se desarrolla las actividades turísticas (Juárez \& Cebrián, 2016). La innovación en el Turismo es primordial debido a la existencia de las necesidades en destacar frente a los competidores, además se basa de la misma manera los demandantes, es decir, los consumidores de dicho producto, mediante esta situación la innovación consigue la oportunidad de crear nuevos productos y servicios, de tal forma, la innovación y el Turismo se encuentran totalmente vinculadas (Román, 2015). 
Es así, que una de las ventajas del Turismo en la innovación es el sector transversal, diverso y flexible, y que gracias a estos aspectos se encuentra relacionadas con el ocio; llegando a tener un uso pertinente del internet para facilitar a las empresas la obtención de un amplio conocimiento con respecto al mercado turístico, además por los diversos cambios que se ha generado dentro del entorno turístico se ha llegado a la adaptación de los distintos clientes a las nuevas tendencias turísticas y que de una u otra forma se han vuelto dinámicos según el avance de la actividad turística, se afirma que la innovación turística se constituye desde dos puntos de vista (Astudillo \& Prada, 2017).

a) Analiza e interpreta los efectos territoriales de las innovaciones y su consecuencia sobre el sistema productivo.

b) Interpreta la renovación de los destinos turísticos.

La innovación es una de las herramientas que se destaca en el desarrollo del turismo, implica que el sector turístico busque la facilidad de crear nuevos productos implementando estrategias eficientes de los recursos tangibles e intangibles del Turismo, la innovación va más allá de los inventos y adopción de bienes y servicios, por lo tanto, la innovación es una forma de pensar y orientar al turismo hacia una nueva gestión (Delgado, Vargas, Montes \& Rodríguez, 2016). Y uno de los modelos que se ha estudiado para el desarrollo del Turismo es la innovación en el emprendimiento que ayuda a los individuos a crear sus propios negocios (Vélez \& Ortiz, 2016).

La innovación en el emprendimiento se ha logrado determinar una nueva manera de gestionar los negocios, pues debido al emprendimiento que trata de buscar, pensar y analizar un espacio para incrementar la fortuna del trabajo está conexo con la innovación al generar ideas innovadoras que resalten con acogimiento al público y logren su diferencia ante sus competidores, es decir, su producto pensado con anterioridad para abrir su negocio, pero a su vez, a través de la innovación se transforma en un producto totalmente mejorado superando las expectativas de los clientes (García, 2014). Siendo así, que si un emprendedor olvida o deja de lado su creatividad de innovación simplemente no puede emprender brindando solamente un producto o servicio común, llegando a ser una rutina en donde se centra en recibir dinero a cambio de lo ofertado, ocasionando el desagrado de adquirir nuevamente el producto, culminando con la minimización de superación de las expectativas del cliente (Schnarch, 2014).

Dentro del emprendimiento las personas identifican la parte más importante, utilizando así, sus capacidades, aptitudes y destrezas para poder ser un recurso indispensable en cuanto a la generación de productos o servicios innovadores, por lo que, no puede competir con una máquina que no introduce nuevas ideas, pues a diferencia del ser humano que sí se encuentra en capacidad de hacerlo (Molina, López \& Contreras, 2014). Es así que la capacidad de innovar es fundamental para el proceso de desarrollo del emprendimiento llegando a crear nuevos y mejores productos, pero, sobre todo, captar momentos óptimos que ayuden al progreso del mismo, implantando situaciones favorables para el beneficio oportuno de uno mismo a través de acciones que incluyan el desarrollo insuperable al frente de otras empresas, para así mantener el equilibrio deseado y esperado (Asprilla, Gutiérrez \& Gutiérrez, 2014). La capacidad de innovación paso a paso llega a ser un gestor determinante en donde predomina la acción a incorporar ideas con tendencias innovadoras a través del pensamiento y comportamiento que realiza una persona al hacer actividades distintas con la finalidad de lograr un producto propio e innovador, tomando en cuenta como factor primordial el desenvolvimiento constante en el entorno cotidiano para el alcance de una labor 
excelente, dejando a un lado lo inservible y acogerse a lo que en verdad va a ser de apoyo principal en dicho pensamiento y creación, lo cual va a ser dirigido al mercado que lo necesite (Grañó, 2019).

\section{Capacidad de Innovación}

A través del tiempo la capacidad de innovación ha ido cambiando gradualmente ya que a partir de ello, se ha descubierto que está compuesta por varias fases que determinan cuán importante es necesario intervenir dentro del tema, debido a sus distintos perfiles y maneras de ver desde diferentes puntos de vista, conllevando a la obtención de conocimientos duraderos y precisos (Saunila, 2014). De esta manera se visualiza la situación acorde a los acontecimientos futuros logrando interpretar y solucionar de la forma más eficaz y eficientemente manteniendo la innovación por delante de los demás y perdurando en el tiempo según la tendencia a surgir; simplemente es un medio que conforme se va transformando, esta a su vez lo hace consigo misma determinando su propia evolución (Ruiz, Ortega, Haro \& Roldán, 2014).

De hecho, se ha introducido varias hipótesis en donde la capacidad de innovación viene dada por el factor cognoscitivo pues mediante esto, se puede aprovechar las distintas ocasiones presentadas en cualquier situación por más mala que sea, conociendo y tratando el ambiente habitual con más facilidad incrementando la confianza por mejorar cada día como persona y empresa a la vez (Lara, Maya \& Maldonado, 2018). Así mismo se centra directa e indirectamente en el incremento de la obtención de ganancias a través de la idea pensada y plasmada en el momento preciso para superarse y encontrarse en la línea perdurable constantemente, de tal forma existe varios materiales y herramientas que aportan con la capacidad de crear nuevas e inimaginables cosas que aumenten el interés del mundo entero mediante personas con un alto grado de innovación que fortifiquen en este ámbito (Chan, Bustamante \& Ordoñez, 2018).

Se ha fijado características que se encuentran relacionadas con el tema como las siguientes: la paciencia ante una situación inesperada, pues aquí perdura la calma como fuente importante para la elección de soluciones correctas y eficaces; estar constantemente involucrado dentro de la situación a acontecer atrapando las buenas ocasiones para el fortalecimiento de lo emprendido; a través de las aptitudes y habilidades de uno mismo, confiar sin dudar al respecto y dar oportunidad a los demás al desenvolvimiento, produce día a día la excelencia; teniendo en cuenta lo anterior es necesario pensar en ideas nuevas y creativas para plasmarlas en el exterior (Castillo, 2018). Por lo antes mencionado, las personas de distinto género tienden a pensar nuevas ideas e implantarlas en ocasiones que creen necesarias, pues cuando se encuentran en el mismo entorno sobresalen con esta acción de forma grupal, a diferencia de un mismo género que se centra en una sola meta, objetivo o simplemente actúa de la misma forma lo cual no favorece dentro del entorno laboral disminuyendo las oportunidades de destacar, sin embargo, las experiencias y habilidades resaltan en cada uno de ellos opacándolo de diferentes maneras al compañero de trabajo dentro de las actividades a desarrollar en el emprendimiento que desea incrementar con mayor rapidez los beneficios (Romero, Montoro \& Garavito, 2017).

\section{La Capacidad de innovación en el género}

La innovación genera progresividad y a la vez competitividad en los distintos emprendimientos a surgir, encontrándose relacionado con el género femenino y masculino, 
que con la unión de los mismos impulsa una nueva manera de cambiar la perspectiva y visión ante los consumidores, tal es el caso que las mujeres con creatividad brindan más alternativas produciendo un desarrollo sobresaliente ante los problemas, siendo la comunicación un medio importante para desarrollar flexibilidad en el emprendimiento; pues los hombres son más persistentes en llegar y cumplir lo deseado, de esta manera aparece el equilibrio de género acompañado de diversas posibilidades de solución y supera los inconvenientes de la mejor forma desempeñando la labor con responsabilidad y organización (Sandoval, 2014). Las mujeres emprendedoras están haciendo contribuciones significativas a sus economías del país a través de sus actividades innovadoras (Aidis, 2016). Las desigualdades de género en el acceso a la educación, el empleo, el emprendimiento, y la creación de innovación reducen el conjunto de talentos para la productividad asignación y, por lo tanto, crecimiento económico (Liberda \& Zajkowska, 2017).

Estudios recientes proporcionan una fuerte evidencia de que la visión ciega de género es una barrera significativa para desarrollar ideas y comportamientos más creativos, esto va más allá de la evaluación de muchas oportunidades de crecimiento (Belghiti-Mahut, Lafont \& Yousfi, 2016). La diversidad de género califica positivamente la relación entre las capacidades de gestión y el rendimiento de la innovación de productos y procesos (RuizJiménez \& del Mar Fuentes-Fuentes, 2016). El género es un factor fundamental dentro de la innovación asociándose de manera positiva y dando resultados fructíferos con un rendimiento aceptable a través de las habilidades y capacidades que poseen cada uno de ellos en la creación de nuevas ideas para resaltar y diferenciarse de los emprendimientos comunes que normalmente se adentran en el mercado competitivo (Garavito, 2017).

\section{Materiales y Métodos}

Se realizó una investigación de tipo documental y de campo, una investigación documental recopila la información a través de varios documentos bibliográficos en relación al tema de interés se valoran por la interpretación en la lectura y crítica, la investigación de campo se realiza mediante un proceso en el cual se obtiene información a partir de la realidad del lugar en cuestión, diagnosticando los problemas y necesidades presentado en dicho lugar (Prieto, 2014). El enfoque de investigación utilizado es cuantitativo con un paradigma deductivo a través de instrumentos de medición (encuestas) se utilizó el constructo Capacidad de Innovación (Selznick \& Mayhew, 2017).

El método de investigación es de carácter no experimental, transversal y descriptivo. No experimental es donde se va analizando la realidad sin manipular los datos después de la observación de la situación, en cuanto a lo transversal por recoger información por una sola vez en un tiempo determinado y fue descriptivo porque se describen las variables para poder especificar los datos más sobresalientes que han sido analizados (Cortes \& Iglesias, 2014).

Para llevar a cabo la investigación se trabajó con el catastro turístico del año 2018 y 2019 del GAD Municipal del cantón Pastaza, el cual representa a una población de 144 emprendedores. Se realizó el muestreo no probabilístico debido que la población tenía una representatividad mínima, por lo cual se aplicó la encuesta a todo el universo del catastro que brindan servicios turísticos como: Alojamiento, Alimentos y Bebidas, Operación e Intermediación y Parques de atracción. La muestra no probabilística es una técnica que depende de las causas y características que el investigador considere para el estudio que se pretende realizar (Otzen \& Manterola, 2017). El instrumento de medición fue sometido a un análisis de confiabilidad de Cronbach para analizar la consistencia interna de los ítems en la 
encuesta, para ello los datos fueron tabulados en el programa estadísticos SPSS. La confiabilidad de Cronbach mide los errores y precisiones que aparecen dentro de una prueba en donde cada pregunta es imprescindible en el instrumento debido a su relación (Espinoza \& Novoa, 2018). La encuesta contiene 42 preguntas y está dividida en 9 dimensiones:

Tabla 1.

Dimensiones de la Capacidad de Innovación

\begin{tabular}{lll}
\hline Dimensiones & Ítems & Concepto Dimensiones \\
\hline Motivación (Mo) & Preguntas $(1,2,3,4,5)$ & $\begin{array}{l}\text { Según Naranjo (2009) la motivación } \\
\text { direcciona e impulsa hacia la perseverancia del } \\
\text { logro de una meta. }\end{array}$ \\
\hline Proactividad (Pro) & Preguntas $(6,7,8,9)$ & $\begin{array}{l}\text { Según Carvajal (2017) señala que es la } \\
\text { capacidad de realizar nuevas cosas, planificar } \\
\text { eventos que tengan iniciativas y ser los } \\
\text { promotores en generar nuevas actividades. }\end{array}$ \\
\hline
\end{tabular}

Auto concepto de innovación (SfC)

Preguntas $(10,11,12,13,14)$

Según el autor Selznick y Mayhew (2018) menciona que es el conjunto de actividades que abarca la creencia en uno mismo para crear nuevas ideas y oportunidades.

Según Selznick y Mayhew (2017) destacó que

Redes (Net) Preguntas $(15,16,17,18)$

Comunicación persuasiva Preguntas $(19,20,21,22,23)$
(Psu) la red es una habilidad social que permite comunicar e interactuar con otras personas.

Según el autor Selznick y Mayhew (2017) determina que la comunicación persuasiva es convencer a alguien de decir o hacer una tarea determinada.

Según Reinosa (2016) mencionó que trabajo en

Trabajo en equipo a través de las diferencias (Tem)

Preguntas $(24,25,26,27)$ equipo es el conjunto de personas que ayudan para obtener un resultado determinado.

Según Selznick y Mayhew (2017) señala que la Cognición creativa es un proceso mental que

Cognición creativa (Crt) Preguntas $(28,29,30,31,32)$ surge de otros procesos mentales, de las vivencias, y del aspecto vital donde se desenvuelve el individuo.

Según García (2012) manifiesta que la intención de innovar es la conversión de conocimientos en nuevos productos $\mathrm{y}$ servicios.

Según Echemendia (2011) destaca que la toma de riesgo es una posibilidad de un resultado generalmente no favorable o de lo esperado.
Toma de riesgo/tolerancia Preguntas

(Rsk) (37,38,39,40,41,42)

Para identificar si existe una diferencia significativa entre hombres y mujeres se realizó una prueba " $t$ " de student con el objetivo de determinar tanto en hombres como en mujeres la comparación entre sus medias. La prueba " $t$ " es un método estadístico deductivo que se realiza para estimar una diferencia significativa entre las medias de dos grupos, se aplica cuando la población tiene una distribución normal y la muestra es pequeña (Sánchez, 2015). 


\section{Resultados}

\section{Confiabilidad de Alfa de Cronbach constructo Capacidad de innovación}

Una vez que se procesaron los datos se pudo comprobar que el Alfa de Cronbach de la variable Capacidad de Innovación tiene aproximadamente un 0.935 lo cual nos permite indicar que es un coeficiente de confiabilidad excelente.

Tabla 2.

Estadística de confiabilidad Capacidad de Innovación

\begin{tabular}{cc} 
Alfa de Cronbach & N de elementos \\
\hline, 935 & 42 \\
\hline Fuente: Elaboración propia
\end{tabular}

\section{Variables Demográficas}

Tabla 3.

Género de la muestra

\begin{tabular}{llcccc}
\hline & Frecuencia & Porcentaje & Porcentaje válido & $\begin{array}{c}\text { Porcentaje } \\
\text { acumulado }\end{array}$ \\
\hline Válido & Femenino & 97 & 67,4 & 67,4 & 67,4 \\
\cline { 2 - 6 } & Masculino & 47 & 32,6 & 32,6 & 100,0 \\
\cline { 2 - 5 } & Total & 144 & 100,0 & 100,0 & \\
\hline \multicolumn{5}{c}{ Fuente: Elaboración propia }
\end{tabular}

Tabla 4.

Edad de la muestra

\begin{tabular}{llcccc}
\hline Género & & Frecuencia & Porcentaje & $\begin{array}{c}\text { Porcentaje } \\
\text { válido }\end{array}$ & $\begin{array}{c}\text { Porcentaje } \\
\text { acumulado }\end{array}$ \\
\hline Femenino & $\mathbf{1 8 - 2 5}$ & 8 & 8,2 & 8,2 & 8,2 \\
& $\mathbf{2 6 - 3 5}$ & 21 & 21,6 & 21,6 & 29,9 \\
& $\mathbf{3 6 - 4 5}$ & 33 & 34,0 & 34,0 & 63,9 \\
& $\mathbf{4 6 - 5 5}$ & 24 & 24,7 & 24,7 & 88,7 \\
& Más de 55 años & 11 & 11,3 & 11,3 & 100,0 \\
\hline Masculino & Total & 97 & 100,0 & 100,0 & 10,6 \\
& $\mathbf{1 8 - 2 5}$ & 5 & 10,6 & 10,6 & 29,8 \\
& $\mathbf{2 6 - 3 5}$ & 9 & 19,1 & 19,1 & 53,2 \\
& $\mathbf{3 6 - 4 5}$ & 11 & 23,4 & 23,4 & 87,2 \\
& $\mathbf{4 6 - 5 5}$ & 16 & 34,0 & 34,0 & 100,0 \\
& Más de 55 años & 6 & 12,8 & 12,8 & 100,0 \\
\hline
\end{tabular}

Fuente: Elaboración propia

Tabla 5.

Escolaridad de la muestra

\begin{tabular}{cclcccc}
\hline Género & & Frecuencia & Porcentaje & $\begin{array}{c}\text { Porcentaje } \\
\text { válido }\end{array}$ & $\begin{array}{c}\text { Porcentaje } \\
\text { acumulado }\end{array}$ \\
\hline Femenino & Válido & Ninguno & 1 & 1,0 & 1,1 & 1,1 \\
& & Primaria & 4 & 4,1 & 4,2 & 5,3 \\
& & Bachillerato & 51 & 52,6 & 53,7 & 58,9
\end{tabular}

Esta obra se comparte bajo la licencia Creative Common Atribución-No Comercial 4.0 International (CC BY-NC 4.0) 


\begin{tabular}{|c|c|c|c|c|c|c|}
\hline \multirow[t]{6}{*}{ Género } & & & Frecuencia & Porcentaje & $\begin{array}{c}\text { Porcentaje } \\
\text { válido }\end{array}$ & $\begin{array}{l}\text { Porcentaje } \\
\text { acumulado }\end{array}$ \\
\hline & & Tercer nivel & 35 & 36,1 & 36,8 & 95,8 \\
\hline & & Cuarto nivel & 4 & 4,1 & 4,2 & 100,0 \\
\hline & & Total & 95 & 97,9 & 100,0 & \\
\hline & Perdidos & Sistema & 2 & 2,1 & & \\
\hline & Total & & 97 & 100,0 & & \\
\hline \multirow[t]{7}{*}{ Masculino } & Válido & Primaria & 1 & 2,1 & 2,2 & 2,2 \\
\hline & & Bachillerato & 18 & 38,3 & 39,1 & 41,3 \\
\hline & & Tercer nivel & 25 & 53,2 & 54,3 & 95,7 \\
\hline & & Cuarto nivel & 2 & 4,3 & 4,3 & 100,0 \\
\hline & & Total & 46 & 97,9 & 100,0 & \\
\hline & Perdidos & Sistema & 1 & 2,1 & & \\
\hline & Total & & 47 & 100,0 & & \\
\hline
\end{tabular}

Fuente: Elaboración propia

Tabla 6.

Clasificación de género del tipo de servicio turístico ofertado

\begin{tabular}{|c|c|c|c|c|c|}
\hline Género & & Frecuencia & Porcentaje & $\begin{array}{l}\text { Porcentaje } \\
\text { válido }\end{array}$ & $\begin{array}{l}\text { Porcentaje } \\
\text { acumulado }\end{array}$ \\
\hline \multirow[t]{5}{*}{ Femenino } & Alojamiento & 30 & 30,9 & 30,9 & 30,9 \\
\hline & Alimentos y Bebidas & 60 & 61,9 & 61,9 & 92,8 \\
\hline & $\begin{array}{l}\text { Operación e } \\
\text { Intermediación }\end{array}$ & 4 & 4,1 & 4,1 & 96,9 \\
\hline & Parques de atracción & 3 & 3,1 & 3,1 & 100,0 \\
\hline & Total & 97 & 100,0 & 100,0 & \\
\hline \multirow[t]{5}{*}{ Masculino } & Alojamiento & 14 & 29,8 & 29,8 & 29,8 \\
\hline & Alimentos y Bebidas & 29 & 61,7 & 61,7 & 91,5 \\
\hline & $\begin{array}{l}\text { Operación e } \\
\text { Intermediación }\end{array}$ & 3 & 6,4 & 6,4 & 97,9 \\
\hline & Parques de atracción & 1 & 2,1 & 2,1 & 100,0 \\
\hline & Total & 47 & 100,0 & 100,0 & \\
\hline
\end{tabular}

Fuente: Elaboración propia

Tabla 7.

Ingresos mensuales de su negocio

\begin{tabular}{|c|c|c|c|c|c|c|}
\hline Género & & & Frecuencia & Porcentaje & $\begin{array}{c}\text { Porcentaje } \\
\text { válido }\end{array}$ & $\begin{array}{l}\text { Porcentaje } \\
\text { acumulado }\end{array}$ \\
\hline \multirow[t]{6}{*}{ Femenino } & Válido & Menos de 500 \$ & 15 & 15,5 & 15,5 & 15,5 \\
\hline & & 501 a $1000 \$$ & 24 & 24,7 & 24,7 & 40,2 \\
\hline & & 1001 a 1500 \$ & 20 & 20,6 & 20,6 & 60,8 \\
\hline & & 1501 a $2000 \$$ & 24 & 24,7 & 24,7 & 85,6 \\
\hline & & Mayor a $2000 \$$ & 14 & 14,4 & 14,4 & 100,0 \\
\hline & & Total & 97 & 100,0 & 100,0 & \\
\hline \multirow[t]{8}{*}{ Masculino } & Válido & Menos de 500 \$ & 6 & 12,8 & 13,0 & 13,0 \\
\hline & & 501 a $1000 \$$ & 14 & 29,8 & 30,4 & 43,5 \\
\hline & & 1001 a $1500 \$$ & 7 & 14,9 & 15,2 & 58,7 \\
\hline & & 1501 a $2000 \$$ & 12 & 25,5 & 26,1 & 84,8 \\
\hline & & Mayor a $2000 \$$ & 7 & 14,9 & 15,2 & 100,0 \\
\hline & & Total & 46 & 97,9 & 100,0 & \\
\hline & Perdidos & Sistema & 1 & 2,1 & & \\
\hline & Total & & 47 & 100,0 & & \\
\hline
\end{tabular}

Fuente: Elaboración propia 
Tabla 81.

Procedencia del emprendedor

\begin{tabular}{llcccc}
\hline Género & Frecuencia & Porcentaje & $\begin{array}{c}\text { Porcentaje } \\
\text { válido }\end{array}$ & $\begin{array}{c}\text { Porcentaje } \\
\text { acumulado }\end{array}$ \\
\hline Femenino & Nacional & 94 & 96,9 & 96,9 & 96,9 \\
& Extranjero & 3 & 3,1 & 3,1 & 100,0 \\
Masculino & Total & 97 & 100,0 & 100,0 & \\
& Nacional & 46 & 97,9 & 97,9 & 97,9 \\
& Extranjero & 1 & 2,1 & 2,1 & 100,0 \\
\hline
\end{tabular}

Fuente: Elaboración propia

Tabla 92.

Contacto con un centro / organismo de apoyo a emprendedores.

\begin{tabular}{llcccc}
\hline Género & & Frecuencia & Porcentaje & $\begin{array}{c}\text { Porcentaje } \\
\text { válido }\end{array}$ & $\begin{array}{c}\text { Porcentaje } \\
\text { acumulado }\end{array}$ \\
\hline Femenino & $\mathrm{Si}$ & 36 & 37,1 & 37,1 & 37,1 \\
& $\mathrm{No}$ & 61 & 62,9 & 62,9 & 100,0 \\
& Total & 97 & 100,0 & 100,0 & 48,9 \\
& $\mathrm{Si}$ & 23 & 48,9 & 48,9 & 100,0 \\
& No & 24 & 51,1 & 51,1 & \\
\hline
\end{tabular}

Fuente: Elaboración propia

Tabla 10.

Tiempo de apertura del negocio

\begin{tabular}{llllll} 
Género & & Frecuencia & & $\begin{array}{c}\text { Porcentaje } \\
\text { válido }\end{array}$ & \multicolumn{1}{c}{$\begin{array}{c}\text { Porcentaje } \\
\text { acumulado }\end{array}$} \\
\hline Femenino & Menor a 1 año & 7 & 7,2 & 7,2 & 7,2 \\
& Entre 1 a 5 años & 28 & 28,9 & 28,9 & 36,1 \\
& Entre 5 a 10 años & 33 & 34,0 & 34,0 & 70,1 \\
& Mayor de 10 años & 29 & 29,9 & 29,9 & 100,0 \\
& Total & 97 & 100,0 & 100,0 & \\
\hline Masculino & Menor a 1 año & 2 & 4,3 & 4,3 & 4,3 \\
& Entre 1 a 5 años & 14 & 29,8 & 29,8 & 34,0 \\
& Entre 5 a 10 años & 16 & 34,0 & 34,0 & 68,1 \\
& Mayor de 10 años & 15 & 31,9 & 31,9 & 100,0 \\
& Total & 47 & 100,0 & 100,0 & \\
\hline
\end{tabular}

Fuente: Elaboración propia

Análisis de las dimensiones de la variable Capacidad de Innovación

Tabla 11.

Análisis de la dimensión motivación

\begin{tabular}{ccccc}
\hline Motivación & $\begin{array}{c}\text { Muy } \\
\text { desfavorable }\end{array}$ & Desfavorable & Favorable & Muy favorable \\
\hline Masculino & & $5,15 \%$ & $40,21 \%$ & $54,64 \%$ \\
\hline Femenino & & $4,26 \%$ & 36,17 & $59,57 \%$ \\
\hline
\end{tabular}

Fuente: Elaboración propia 
Tabla 12.

Análisis de la dimensión proactividad

\begin{tabular}{ccccc}
\hline Proactividad & $\begin{array}{c}\text { Muy } \\
\text { desfavorable }\end{array}$ & Desfavorable & Favorable & Muy favorable \\
\hline Masculino & & $6,36 \%$ & $40,43 \%$ & $53,19 \%$ \\
\hline Femenino & $1,03 \%$ & $4,12 \%$ & $40,21 \%$ & $54,64 \%$ \\
\hline \multicolumn{5}{c}{ Fuente: Elaboración propia }
\end{tabular}

Tabla 13.

Análisis de la dimensión auto concepto de innovación

\begin{tabular}{ccccc}
\hline $\begin{array}{c}\text { Auto concepto } \\
\text { innovación }\end{array}$ & $\begin{array}{c}\text { Muy } \\
\text { desfavorable }\end{array}$ & Desfavorable & Favorable & Muy favorable \\
\hline Masculino & $4,26 \%$ & $4,26 \%$ & $36,17 \%$ & $55,32 \%$ \\
\hline Femenino & $1,03 \%$ & $4,12 \%$ & $39,18 \%$ & $55,67 \%$ \\
\hline \multicolumn{5}{c}{ Fuente: Elaboración propia }
\end{tabular}

Tabla 14.

Análisis de la dimensión redes

\begin{tabular}{ccccc}
\hline Redes & $\begin{array}{c}\text { Muy } \\
\text { desfavorable }\end{array}$ & Desfavorable & Favorable & Muy favorable \\
\hline Masculino & $6,38 \%$ & $8,51 \%$ & $59,57 \%$ & $25,53 \%$ \\
\hline Femenino & $2,06 \%$ & $11,34 \%$ & $40,21 \%$ & $46,39 \%$ \\
\hline \multicolumn{5}{c}{ Fuente: Elaboración propia } \\
\hline
\end{tabular}

Tabla 15.

Análisis de la dimensión Comunicación persuasiva

\begin{tabular}{ccccc}
\hline $\begin{array}{c}\text { Comunicación } \\
\text { persuasiva }\end{array}$ & $\begin{array}{c}\text { Muy } \\
\text { desfavorable }\end{array}$ & Desfavorable & Favorable & Muy favorable \\
\hline Masculino & $2,13 \%$ & $8,51 \%$ & $53,19 \%$ & $36,17 \%$ \\
\hline Femenino & $1,03 \%$ & $14,43 \%$ & $42,27 \%$ & $42,27 \%$ \\
\hline \multicolumn{5}{c}{ Fuente: Elaboración propia }
\end{tabular}

Fuente: Elaboración propia

Tabla 16.

Análisis de la dimensión Trabajo en equipo

\begin{tabular}{ccccc}
\hline $\begin{array}{c}\text { Trabajo en } \\
\text { equipo }\end{array}$ & $\begin{array}{c}\text { Muy } \\
\text { desfavorable }\end{array}$ & Desfavorable & Favorable & Muy favorable \\
\hline Masculino & & $8,51 \%$ & $40,43 \%$ & $51,06 \%$ \\
\hline Femenino & & $5,15 \%$ & $48,45 \%$ & $46,39 \%$ \\
\hline
\end{tabular}

Fuente: Elaboración propia

Tabla 17.

Análisis de la dimensión Cognición creativa

\begin{tabular}{ccccc}
\hline $\begin{array}{c}\text { Cognición } \\
\text { creativa }\end{array}$ & $\begin{array}{c}\text { Muy } \\
\text { desfavorable }\end{array}$ & Desfavorable & Favorable & Muy favorable \\
\hline Masculino & & $2,13 \%$ & $38,30 \%$ & $58,57 \%$ \\
\hline Femenino & & $3,09 \%$ & $30,93 \%$ & $65,98 \%$ \\
\hline
\end{tabular}

Fuente: Elaboración propia

Tabla 18.

Análisis de la dimensión Intención de innovar

\begin{tabular}{ccccc}
\hline $\begin{array}{c}\text { Intención de } \\
\text { innovar }\end{array}$ & $\begin{array}{c}\text { Muy } \\
\text { desfavorable }\end{array}$ & Desfavorable & Favorable & Muy favorable \\
\hline Masculino & & $4,26 \%$ & $46,81 \%$ & $48,94 \%$ \\
\hline Femenino & $1,03 \%$ & $3,09 \%$ & $48,45 \%$ & $47,42 \%$ \\
\hline \multicolumn{5}{c}{ Fuente: Elaboración propia }
\end{tabular}


Tabla 19.

Análisis de la dimensión Toma de riesgos

\begin{tabular}{|c|c|c|c|c|}
\hline Toma de riesgos & $\begin{array}{c}\text { Muy } \\
\text { desfavorable }\end{array}$ & Desfavorable & Favorable & Muy favorable \\
\hline Masculino & $2,13 \%$ & $6,38 \%$ & $70,21 \%$ & $21,28 \%$ \\
\hline Femenino & & $13,40 \%$ & $56,70 \%$ & $29,90 \%$ \\
\hline
\end{tabular}

Análisis diferencia de medias entre hombres y mujeres

Tabla 20.

Diferencia de medias entre hombres y mujeres (prueba $t$ student)

\begin{tabular}{lccc}
\hline $\begin{array}{l}\text { Capacidades } \\
\text { innovadoras }\end{array}$ & $\mathbf{t}$ & gl & Sig. (bilateral) \\
\hline $\begin{array}{l}\text { Se asumen varianzas } \\
\text { iguales }\end{array}$ & 0,588 & 141 & 0,557 \\
$\begin{array}{l}\text { No se asumen } \\
\text { varianzas iguales }\end{array}$ & 0,580 & 85,394 & 0,563 \\
\hline
\end{tabular}

La prueba $t$ de student indica que no existen diferencias significativas entre los hombres y mujeres de la muestra

\section{Conclusiones}

Con la investigación realizada se concluye que en la región amazónica del Ecuador, específicamente en el cantón Pastaza, existe una predominancia del género femenino con un $67 \%$ comparados con el masculino con un $33 \%$, los emprendedores se centran entre los 26 a 55 años presentando una población joven adulta, en su gran mayoría son casados y en unión libre, su nivel de estudio indica que han llegado a tener una educación secundaria que les ha permitido abrir negocios propios con ingresos económicos que le permiten generar una estabilidad en su familia, a pesar que no han estado en contacto con organismos de apoyo a emprendedores, la apertura de sus emprendimientos está entre uno a más de diez años en el mercado y su procedencia es local. Mediante el análisis de las dimensiones de la encuesta Capacidad de Innovación se evidenció una situación favorable de los emprendedores del sector turístico demostrando que poseen habilidades para desarrollar actividades con voluntad e ingenio dentro del entorno turístico a través de circunstancias que les permitan gestionar e identificar oportunidades en el mercado actual en concordancia con la iniciativa, trabajo en equipo, persistencia, experimentación de nuevas cosas e intención propicia de cada uno de ellos; sin embargo, existe un porcentaje mínimo de negativismo en cuanto a la comunicación con más personas, convencimiento en la solución añadida y el miedo a tomar decisiones que cambien su situación, afectando de una u otra manera la relación con los consumidores e ingresos a los sitios de emprendimiento.

El nivel de capacidad de innovación tanto en mujeres como en hombres emprendedores no presenta diferencias significativas según los resultados obtenidos a través de la prueba $t$ de student $(p \geq 0,05)$. Estos resultados abren nuevas líneas de investigación para analizar si existen relaciones entre la capacidad de emprendimiento, la intención y la orientación emprendedora con las habilidades y capacidades de innovación, de la misma manera, se pueden desarrollar investigaciones que tengan como variables de estudio analizar aspectos demográficos como la edad o la influencia del entorno familiar en el campo de la Esta obra se comparte bajo la licencia Creative Common Atribución-No Comercial 4.0 International (CC BY-NC 4.0) 
innovación y el emprendimiento. La aplicación de este modelo de investigación en otros contextos y regiones a nivel mundial permitirá generar información que corrobore los resultados obtenidos o en su defecto estar sujeto a una posible falsación, con lo cual se aportará al conocimiento científico del emprendimiento y la innovación.

\section{Bibliografía}

Aidis, R. (2016). Three faces of innovation: institutions, gender and entrepreneurship in Latin America. In Research Handbook on Gender and Innovation. Edward Elgar Publishing.

Asprilla, E., Gutiérrez , J. M., \& Gutiérrez , J. A. (2014). Emprendimiento e investigación en la escala de la formación profesional y la innovación empresarial en Colombia. Escuela de Administración de Negocios, 76. Obtenido de https://www.redalyc.org/pdf/206/20631321008.pdf

Álvarez, P., Andrade, M., Bravo, J., Rodríguez, M., \& José, G. (2016). La Innovacion y el Emprendimiento: necesidades en la educacion superior. Dialnet, 4(7). Obtenido de file://C:/Users/hp/Downloads/Dialnet-LaInnovacionYElEmprendimiento6667028.pdf

Arenas, R. (2017). Análisis dinámico de la innovación abierta ¿Efecto sustitución o complementariedad? (Tesis doctoral, Universidad Politécnica de Valencia). Obtenido de https://riunet.upv.es/bitstream/handle/10251/86218/ARENAS\%20$\% 20 \mathrm{An} \% \mathrm{C} 3 \%$ A1lisis\%20din\%C3\%A1mico\%20de\%20la\%20innovaci\%C3\%B3n\% 20abierta\%20\%C2\%BFefecto\%20sustituci\%C3\%B3n\%20o\%20complementariedad $\% 3 F E v i d e . . . . p d f ?$ sequence $=1$

Astudillo, S., \& Prada, J. (2017). Empresas turísticas y factores de innovación: Evidencias a partir del caso de estudio de Cuenca (Ecuador). Redalyc, 17(3). Obtenido de https://www.redalyc.org/pdf/1154/115454650001.pdf

Bembibre, C. (2011). Importancia del Turismo. Obtenido de https://www.importancia.org/turismo.php

Belghiti-Mahut, S., Lafont, A. L., \& Yousfi, O. (2016). Gender gap in innovation: a confused link?. Journal of Innovation Economics Management, (1), 159-177.

Carvajal, P. (2017). Personalidad Proactiva y Capacidad emprendedora. (Tesis de grado, Universidad Cesar Vallejo, Lima. Obtenido de http://repositorio.ucv.edu.pe/bitstream/handle/UCV/3195/Carbajal_SPL.pdf?sequenc $\mathrm{e}=1 \&$ is Allowed $=\mathrm{y}$

Castillo, L. (2018). Capacidad emprendedora y resilencia como emprendedores sobre el potencial emprendedor emprearial. (Tesis de grado, Universidad Privada de Lima). Obtenido de repositorioacademico.usmp.edu.pe: http://www.repositorioacademico.usmp.edu.pe/bitstream/usmp/4361/1/castillo_rly.p df

Chan, T., Bustamante, S., \& Ordoñez, M. (2018). Análisis de la capacidad de internacionalización de los negocios emprendedores de la ciudad de Guayaquil. Scielo, 10(5). Obtenido de scielo.sld.cu: http://scielo.sld.cu/scielo.php?pid=S221836202018000500169\&script=sci_arttext\&tlng=en

Cortes, M., \& Iglesias, M. (2014). Generalidades sobre Metodología. Obtenido de http://www.unacar.mx/contenido/gaceta/ediciones/metodologia_investigacion.pdf

Delgado, A., Vargas, E., Montes, J., \& Rodríguez, F. (2016). Innovation in tourism companies, where are they and where are de going? An approach to the state of 
knowledge. $\quad$ Science, $12(4)$ Obtenido de https://upcommons.upc.edu/bitstream/handle/2117/96575/778-3792-1-PB.pdf

Echemedía, B. (2011). Definicón de riesgo y sus implicaciones. Scielo, 49(3). Obtenido de http://scielo.sld.cu/scielo.php?script=sci_arttext\&pid=S1561-30032011000300014

Espinoza, S. C., \& Muñoz, F. N. (2018). Ventajas del alfa ordinal respecto al alfa de Cronbach ilustradas con la encuesta AUDIT-OMS. Scielo, 42. Obtenido de https://www.scielosp.org/pdf/rpsp/2018.v42/e65/es

Garavito Hernández , Y. (2017). La innovación de producto: un análisis de sus determinantes y su efecto en la supervivencia empresarial. (Tesis doctoral, Universidad Complutense de Madrid). Obtenido de eprints.ucm.es: https://eprints.ucm.es/40718/1/T38202.pdf

García, F. (2012). Concepto Innovación. Obtenido de https://www.acofi.edu.co/wpcontent/uploads/2013/08/DOC_PE_Conceptos_Innovacion.pdf

García Arango, G. A. (2014). Bioética, emprendimiento e innovación en el contexto latinoamericano. Redalyc, 11(1). Obtenido de redalyc.org: https://www.redalyc.org/pdf/695/69531554014.pdf

Gobierno Provincial de Pastaza. (2019). Informacion de la Provincia de Pastaza. Obtenido de https://pastaza.gob.ec/pastaza/informacion/informacion-de-la-provincia-depastaza/

Grañó, M. (2019). La capacidad de innovación es una cuestión de supervivencia. Obtenido de http://www.rrhhdigital.com/editorial/134953/La-capacidad-de-innovacion-es-una-cuestion-de-supervivencia

Juarez, L. O., \& Cebrian, R. G. (2016). Estructura del mercado turistico. Paraninfo. Obtenido de

https://books.google.es/books?hl=es\&lr=\&id=mfj_CwAAQBAJ\&oi=fnd\&pg=PR1

$\& d q=$ turismo+en+el+mundo $+\mathrm{a} \% \mathrm{C} 3 \% \mathrm{~B} 1 \mathrm{o}+2016 \& \mathrm{ots}=0 \mathrm{a} 3 \mathrm{vHvosFL} \& \mathrm{sig}=0-$ _p6PmBaX3cTw5qNoJAyR0Qe8Y\#v=onepage \&q=turismo\%20en\%20el\%20mund o\%20a\%C3\%B10\%202016\&f=false

Lara , G., Maya , A., \& Maldonado , B. (2018). Actividad emprendedora y competitividad en el Ecuador. Global de negocios, 6(1). Obtenido de poseidon01.ssrn.com: file://C:/Users/hp/Downloads/SSRN-id3041453.pdf

Liévano, J., Vargas, P., Pico, B., \& Perez, J. (2018). Importancia de innovación para los emprendedores de micro y pequeñas empresas mexicanas del sector servicios en Villahermosa-Tabasco y Mexicali Baja California. Estudio cuantitativo. European Scientific Journal, 14(22). Obtenido de eujournal.org: https://eujournal.org/index.php/esj/article/view/11121/10659

Liberda, B., \& Zajkowska, O. (2017). Innovation and Entrepreneurship Policies and Gender Equity. International Journal of Contemporary Management, 16 (1)), 37-59.

López, R., Palomeque, J., \& Fadul, J. (2016). Diagnóstico Ecológico en la determinación de los destinos en la Provincia El Oro, Ecuador. Scielo, 8(3). Obtenido de http://scielo.sld.cu/scielo.php?script=sci_arttext\&pid=S2218-36202016000300015

Molina, R., López , A., \& Contreras , R. (2014). El emprendimiento y crecimiento de la pymes. Redalyc, 24(1). Obtenido de redalyc: https://www.redalyc.org/pdf/416/41648308006.pdf

Naranjo, M. (2009). Motivación, perspectivas teóricas y algunas consideraciones de su importancia en el ambito educativo. Redalyc, 33(2). Obtenido de https://www.redalyc.org/pdf/440/44012058010.pdf 
Otsen, T., \& Manterola, C. (2017). tecnicas de Muestreo a una población de estudio. Scielo, 1(35). Obtenido de https://scielo.conicyt.cl/pdf/ijmorphol/v35n1/art37.pdf

Prieto, A. (2014). Master en Ciencia de Datos e Ingeniería de Computacion. Obtenido de http://atc.ugr.es/pages/personal/propia/alberto_prieto/conferencias_pdfs/investigacio n_cientifica_a_prieto/\% 21

Putruele, M. (2017). Innovación turística: las estrategias para traer más viajeros a la ciudad. Obtenido de https://www.infobae.com/turismo/2017/06/27/innovacion-turistica-lasestrategias-para-atraer-mas-viajeros-a-la-ciudad/

Ramírez, J., \& Vega, O. (2015). Sistema de Informacion gerencial e innovacion para el desarrollo de las organizaciones. Télématique, 14(2). Obtenido de file:///C:/Users/hp/Downloads/220-Article\%20Text-224-1-10-20190718.pdf

Reinosa, E. (2016). Trabajo en equipo. Reflexiones para un mejor desempeño. Obtenido de https://www.aacademica.org/ern/6.pdf

Restrepo, A. (2013). Innovacion, Concepto y Retos en la Agenda Universitaria. Estudios de derecho, 70(156). Obtenido de file:///E:/material\%202/Innovación\%20y\%20emprendimiento\%20en\%20el\%20secto r\%20turistico/INNOVACIÓN\%20CONCEPTO\%20Y\%20RETOS\%20EN\%20LA\% 20AGENDA\%20UNIVERSITARIA.pdf

Román, L. (2015). Innovacion y Turismo: El Producto Turistico Frances. (Tesis de grado. Universidad de Valladolid), Segovia. Obtenido de http://uvadoc.uva.es/bitstream/handle/10324/13800/TFGN.277.pdf;jsessionid=4F1045B75BEC6F3BD3DE46CDE4E0C972?sequence $=1$

Romero, A., Montoro, Á., \& Garavito, Y. (2017). El efecto de la diversidad de género y el nuvel educativo en la innovación. Scielo, 57(2). Obtenido de scielo.br: http://www.scielo.br/pdf/rae/v57n2/0034-7590-rae-57-02-0123.pdf

Ruiz, A., Ortega, T., Haro, C., \& Roldán, M. (2014). El proceso de co-creación de valor y su impacto en la estrategia de innovación en empresas de servicio. Redalyc, 10(2). Obtenido de redalyc.org: https://www.redalyc.org/pdf/549/54930454004.pdf

Ruiz-Jiménez, J. M., \& del Mar Fuentes-Fuentes, M. (2016). Management capabilities, innovation, and gender diversity in the top management team: An empirical analysis in technology-based SMEs. BRQ Business Research Quarterly, 19(2), 107-121.

Samson, E. (2015). Turismo Sostenible un nuevo motor para el desarrollo del Ecuador. Obtenido de https://www.usfq.edu.ec/publicaciones/enfoque/Documents/enfoque_018.pdf

Sánchez, R. (2015). t-de Student. Uso y abusos. Scielo, 26(1). Obtenido de http://www.scielo.org.mx/scielo.php?script=sci_arttext\&pid=s018821982015000100009

Sandoval, A. (2014). Diversidad de género como determinate de la innovación. Obtenido de https://pdfs.semanticscholar.org/d041/8ccdba027d3bd42473607407464197540161.p df

Saunila, M. (2014). Capacidad de innovación. Obtenido de file:///C:/Users/Usuario/Downloads/Minna-2014-Capacidad-deInnovaci\%C3\%B3n.pdf

Schnarch, A. (2014). emprendimiento exitoso. Obtenido de https://books.google.es/books?hl=es\&lr=\&id=X8O4DQAAQBAJ\&oi=fnd\&pg=PT5 $\& \mathrm{dq}=$ potencial+innovador+emprendedores+cubanos\&ots $=q \mathrm{TU} \mathrm{pfSq} 4 \mathrm{rD} \& \operatorname{sig}=\mathrm{kD} 9 \mathrm{R}$ A6Mw8BUPMwcagrUukIsMuYk\#v=onepage \&q\&f=false

Selznik, B., \& Mayhew, M. (2017). Measuring Undergraduates Innovacion Capacities. CrossMark, 59.

Obtenido de 
file:///E:/material\%202/Innovación\%20y\%20emprendimiento\%20en\%20el\%20secto r\%20turistico/Capacidda\%20de\%20innovacion\%20graduados\%20instrumento.pdf Sustentur, (2015). Innovación, crucial para promover el turismo sostenible. Obtenido de https://sustentur.com.mx/innovacion-para-promover-el-turismo-sostenible/

Velez, X., \& Ortiz, S. (2016). Emprendimiento e innovación: Una aproximación teórica. Dialnet, 2(4), 346-369. Obtenido de dialnet.unirioja.es: https://dialnet.unirioja.es/servlet/articulo?codigo=5802889

\section{Apéndice 1}

Encuesta Capacidad de Innovación

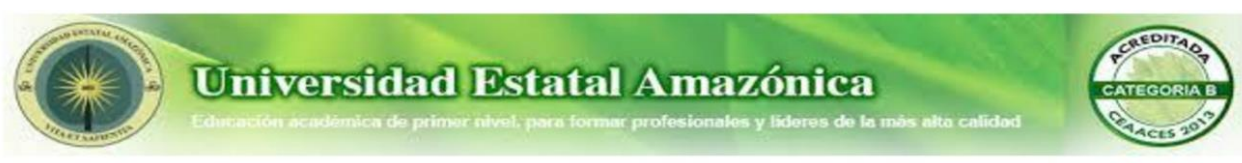

La presente encuesta tiene la finalidad de analizar las capacidades de innovación de los emprendedores turísticos del Cantón Pastaza: Un enfoque de género

Agradecemos por su ayuda en la elaboración de este instrumento de medición. Por favor conteste las preguntas en base al requerimiento que se indica en el instrumento, le tomará un tiempo máximo de 10 minutos y la información será manejada bajo reserva.

Marque con una " $\mathbf{X}$ " la respuesta seleccionada.

Perfil demográfico

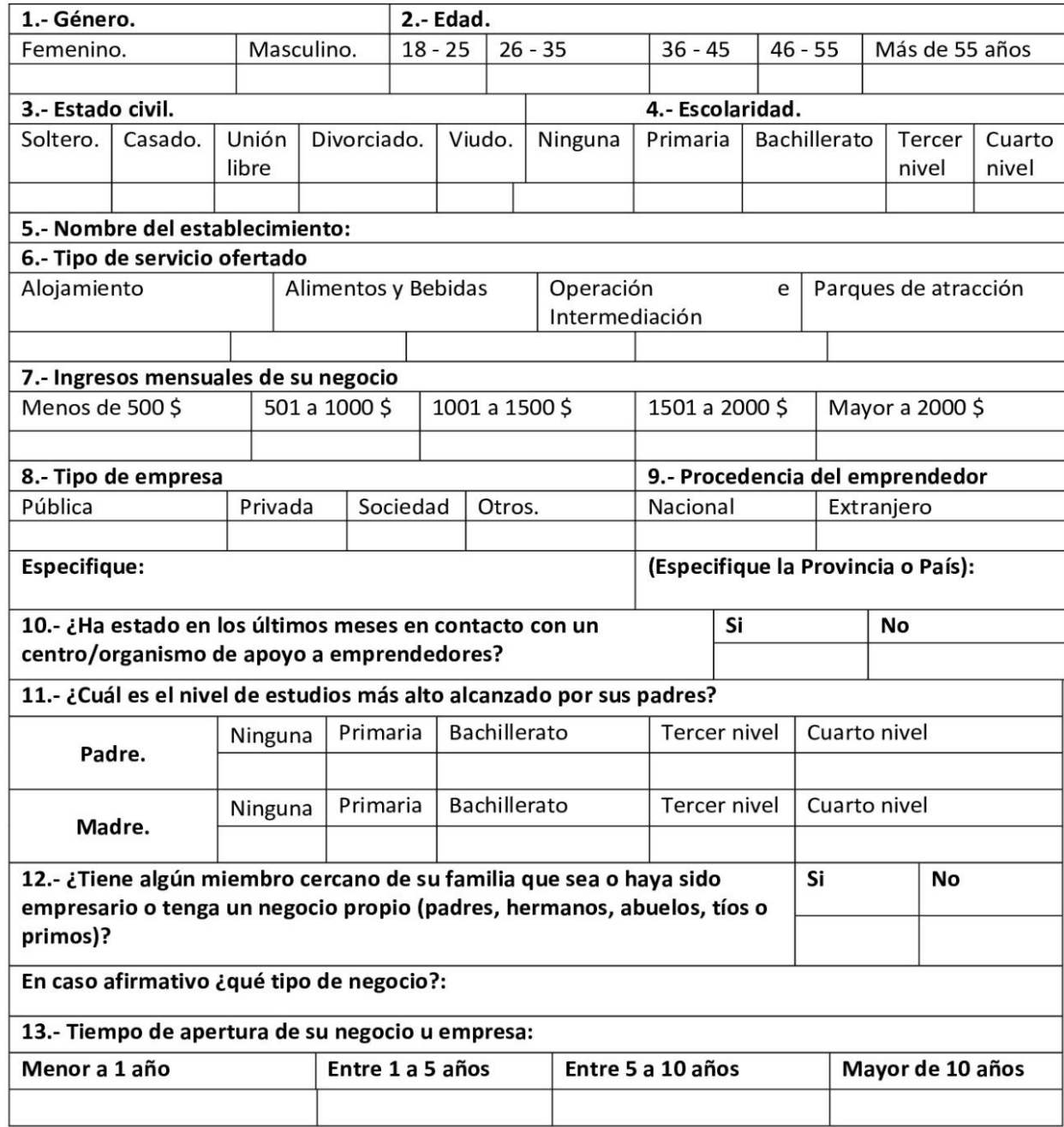

Esta obra se comparte bajo la licencia Creative Common Atribución-No Comercial 4.0 International (CC BY-NC 4.0) Revista de la Universidad Internacional del Ecuador. URL: https://www.uide.edu.ec/ 


\section{Universidad Estatal Amazónica}

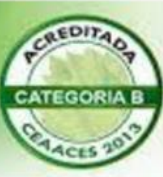

Indique su grado de acuerdo o desacuerdo en una escala del 1 al 5, según los siguientes valores:

\begin{tabular}{|c|c|c|c|c|}
\hline $\mathbf{1}$ & $\mathbf{2}$ & $\mathbf{3}$ & $\mathbf{4}$ & $\mathbf{5}$ \\
\hline $\begin{array}{c}\text { Totalmente en } \\
\text { desacuerdo }\end{array}$ & En desacuerdo & $\begin{array}{c}\text { Ni de acuerdo, ni } \\
\text { en desacuerdo }\end{array}$ & De acuerdo & $\begin{array}{c}\text { Totalmente de } \\
\text { acuerdo }\end{array}$ \\
\hline
\end{tabular}

\begin{tabular}{|c|c|c|c|c|c|c|}
\hline \multicolumn{2}{|r|}{ Motivación (Mo) } & 1 & 2 & 3 & 4 & 5 \\
\hline 1 & $\begin{array}{l}\text { Persisto hacia el logro de objetivos a largo plazo incluso después de } \\
\text { experimentar contratiempos. }\end{array}$ & & & & & \\
\hline 2 & $\begin{array}{l}\text { Respondo eficazmente a los desafíos inesperados que surgen al trabajar en } \\
\text { diversos proyectos o actividades. }\end{array}$ & & & & & \\
\hline 3 & $\begin{array}{l}\text { Persigo un objetivo incluso después de que alguien me haya dicho que será } \\
\text { difícil de lograr. }\end{array}$ & & & & & \\
\hline 4 & Continúo trabajando en un problema hasta que encuentro una solución. & & & & & \\
\hline 5 & $\begin{array}{l}\text { Mantengo el interés en un proyecto o actividad que no tiene una fecha de } \\
\text { finalización clara. }\end{array}$ & & & & & \\
\hline \multicolumn{2}{|r|}{ Proactividad (Pro) } & 1 & 2 & 3 & 4 & 5 \\
\hline 6 & Inicio acciones que cambien positivamente una situación para otros. & & & & & \\
\hline 7 & Inicio acciones que cambien positivamente una situación para mí. & & & & & \\
\hline 8 & Propongo ideas que ayuden a hacer del mundo un lugar mejor. & & & & & \\
\hline 9 & Recopilo información de múltiples fuentes para lograr un objetivo. & & & & & \\
\hline \multicolumn{2}{|r|}{ Auto concepto de innovación (SfC) } & 1 & 2 & 3 & 4 & 5 \\
\hline 10 & Se me ocurre un concepto original que me beneficiará a mí mismo y a otros. & & & & & \\
\hline 11 & Se me ocurre algo nuevo que beneficiará a otros. & & & & & \\
\hline 12 & Otros me han dicho que soy un buen solucionador de problemas. & & & & & \\
\hline 13 & $\begin{array}{l}\text { Por lo general, puedo resolver los problemas que encuentro en mi vida } \\
\text { cotidiana. }\end{array}$ & & & & & \\
\hline 14 & Me veo como un pensador creativo. & & & & & \\
\hline \multicolumn{2}{|r|}{ Redes (Net) } & 1 & 2 & 3 & 4 & 5 \\
\hline 15 & Inicio conversaciones con otras personas que no conozco muy bien. & & & & & \\
\hline 16 & Me presento con nuevas personas de mi rango de edad en un entorno social. & & & & & \\
\hline 17 & Me presento con personas mayores que yo en un entorno social. & & & & & \\
\hline 18 & Convierto una nueva relación en una amistad más cercana. & & & & & \\
\hline \multicolumn{2}{|r|}{ Comunicación Persuasiva (Psu) } & 1 & 2 & 3 & 4 & 5 \\
\hline 19 & $\begin{array}{l}\text { Puedo convencer a un grupo de que tengo la respuesta correcta a un } \\
\text { problema. }\end{array}$ & & & & & \\
\hline 20 & $\begin{array}{l}\text { Puedo convencer a alguien de que mi solución es la correcta para realizar } \\
\text { una tarea determinada. }\end{array}$ & & & & & \\
\hline 21 & Expreso claramente mi visión para resolver problemas a otros. & & & & & \\
\hline 22 & Persuado a otros para que apoyen mi punto de vista. & & & & & \\
\hline 23 & Explico mi razonamiento para tomar decisiones por otros. & & & & & \\
\hline \multicolumn{2}{|r|}{ Trabajo en equipo a través de la diferencia (Tem) } & 1 & 2 & 3 & 4 & 5 \\
\hline 24 & $\begin{array}{l}\text { Trabajo como parte de un grupo con personas que tienen diferentes } \\
\text { habilidades que las mías. }\end{array}$ & & & & & \\
\hline 25 & $\begin{array}{l}\text { Trabajo como parte de un grupo con personas de raza / etnia diferente a la } \\
\text { mía. }\end{array}$ & & & & & \\
\hline 26 & Trabajo en equipo con personas con quienes me encuentro por primera vez. & & & & & \\
\hline 27 & Trabajo como parte de un grupo para lograr un objetivo común. & & & & & \\
\hline
\end{tabular}

Esta obra se comparte bajo la licencia Creative Common Atribución-No Comercial 4.0 International (CC BY-NC 4.0) Revista de la Universidad Internacional del Ecuador. URL: https://www.uide.edu.ec/ 


\section{1. :) Universidad Estatal Amazónica}

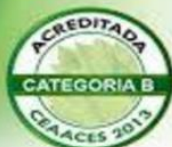

\begin{tabular}{|c|c|c|c|c|c|c|}
\hline \multicolumn{2}{|r|}{ Cognición creativa (Crt) } & \multirow[t]{2}{*}{1} & \multirow[t]{2}{*}{2} & \multirow[t]{2}{*}{3} & \multirow[t]{2}{*}{4} & \multirow[t]{2}{*}{5} \\
\hline 28 & Soy capaz de generar ideas originales. & & & & & \\
\hline 29 & $\begin{array}{l}\text { Me gusta desarrollar nuevas estrategias para ayudar a que las ideas se } \\
\text { conviertan en realidades. }\end{array}$ & & & & & \\
\hline 30 & Disfruto que me pidan ideas nuevas. & & & & & \\
\hline 31 & $\begin{array}{l}\text { Con frecuencia me pregunto "¿Qué podría hacer para mejorar esta } \\
\text { situación?" }\end{array}$ & & & & & \\
\hline 32 & $\begin{array}{l}\text { Me gusta experimentar con diferentes alternativas para completar la misma } \\
\text { tarea. }\end{array}$ & & & & & \\
\hline \multicolumn{2}{|r|}{ Intención de innovar (Int) } & 1 & 2 & 3 & 4 & 5 \\
\hline 33 & $\begin{array}{l}\text { Desarrollo estrategias para dirigir mis esfuerzos y los de otros para alcanzar } \\
\text { nuevas oportunidades (como desarrollar un plan de acción). }\end{array}$ & & & & & \\
\hline 34 & $\begin{array}{l}\text { Identifico nuevas oportunidades (como un nuevo producto o servicio, una } \\
\text { forma más efectiva de realizar tareas, o una nueva forma de resolver un } \\
\text { problema social común). }\end{array}$ & & & & & \\
\hline 35 & $\begin{array}{l}\text { Adquiero los recursos necesarios para aprovechar una nueva oportunidad } \\
\text { (como experiencia, aprobaciones institucionales, financieras). }\end{array}$ & & & & & \\
\hline 36 & $\begin{array}{l}\text { Creo una entidad para aprovechar nuevas oportunidades (por ejemplo, } \\
\text { organizo un equipo, empresa / sin fines de lucro, o un club dedicado a la } \\
\text { nueva oportunidad). }\end{array}$ & & & & & \\
\hline \multicolumn{2}{|r|}{ Toma de riesgos / tolerancia (Rsk) } & 1 & 2 & 3 & 4 & 5 \\
\hline 37 & Sugiero mejoras a la idea de un compañero de trabajo. & & & & & \\
\hline 38 & $\begin{array}{l}\text { Desafío las sugerencias de un compañero de trabajo sobre cómo resolver un } \\
\text { problema. }\end{array}$ & & & & & \\
\hline 39 & $\begin{array}{l}\text { No estoy de acuerdo con el punto de vista de un miembro del personal en } \\
\text { un entorno laboral. }\end{array}$ & & & & & \\
\hline 40 & $\begin{array}{l}\text { Expreso mi opinión, incluso si no estoy seguro de que otros compartan mi } \\
\text { punto de vista. }\end{array}$ & & & & & \\
\hline 41 & $\begin{array}{l}\text { No estoy de acuerdo con el punto de vista de otra persona en un entorno } \\
\text { fuera del trabajo. }\end{array}$ & & & & & \\
\hline 42 & Desafío las sugerencias de una persona sobre cómo resolver un problema. & & & & & \\
\hline
\end{tabular}

\section{¡Gracias por su colaboración!}

